\title{
Conductance of ferromagnetic nanowires
}

\author{
H. Mehrez and S. Ciraci \\ Department of Physics, Bilkent University, Bilkent 06533, Ankara, Turkey
}

(Received 23 February 1998)

\begin{abstract}
The conductance distribution obtained from an ensemble of stretching ferromagnetic nanowires or point contacts do not exhibit peaks near the integer multiples of $2 e^{2} / h$. This observation has been interpreted as the absence of conductance quantization. In this report, we examine various features of electron transport through the Ni nanowires and clarify the behavior of conductance distribution that is different from gold and copper nanowires. Our study concludes that the tunneling through the closely spaced states near $\mathrm{E}_{F}$ that originate from crystal field and spin split $3 d$ states of $\mathrm{Ni}$ prevents the plateaus of conductance from forming in the course of stretch. [S0163-1829(98)07935-1]
\end{abstract}

Recently the electron transport through an atomic size connective neck or point contact created by scanning tunneling microscopy tip has been a subject of active study. ${ }^{1-4}$ The most essential feature of the quantum transport through nano-objects is the level spacing, $\Delta \epsilon=\epsilon_{i+1}-\epsilon_{i}$, of the electronic states quantized in the constriction that is materialized by a connective neck or contact. For a free electron gas $\Delta \epsilon$ $\sim \lambda_{F}^{-2}$. Here, the neck incorporates just a few atoms and has a diameter in the range of $\lambda_{F}(5-10 \AA)$ shortly before the break. Accordingly, the level spacing of nanowires having metallic electron density can be as wide as $1 \mathrm{eV}$. While the constriction atoms that have s-valence electrons (such as $\mathrm{Na}$, $\mathrm{Au})$ generally comply with the above argument, $\Delta \epsilon(\epsilon)$ can be rather irregular and depend strongly on the electronic structure of free atoms ${ }^{3,5,6}$ and local bonding structure. ${ }^{6}$ The quantum nature of electronic structure and its level spacing near $\mathrm{E}_{F}$, with $\Delta \epsilon\left(E_{F}\right)$ is reflected to the measured variation of conductance $G$, with the tip displacement $s$. As argued earlier, the ballistic conduction through each channel due to a current transporting state has the transmission $\mathcal{T} \leqslant 1$ and hence contributes to $G$ by $\sim 2 e^{2} / h$ (including both spins). Wide $\Delta \epsilon$ prevents the staircase structure of $G(s)$ from smearing $^{7}$ (due to channel mixing, finite temperature, bias voltage, and tunneling) and makes flat plateaus more pronounced. The cross section of the neck (or contact) $A$ changes discontinuously with $s$; each time a finite number of atoms disappear from the neck ${ }^{4,8}$ by pulling the tip from the nanoindentation (or conversely new atoms are implemented to the contact ${ }^{9}$ by pushing the tip further to the sample). As predicted earlier, ${ }^{3}$ and confirmed by recent experimental ${ }^{10}$ and theoretical studies, ${ }^{4,8}$ the discontinuous change of $A$ gives rise to sudden jumps of $G(s)$. Various atomic processes that lead to discontinuous variation of $G(s)$ have been revealed from the recent simulations based on the classical molecular-dynamics calculations. ${ }^{4,8,9}$ The limited electronic screening at the neck and various effects originating thereof (such as enhanced polarity, charge transfer, and enhanced dipole and resonant excitations ${ }^{9}$ require further study).

Apart from the sudden jumps and flat plateaus, the histogram distribution of conductance $D(G)$ extracted from an ensemble of the wire-stretching experiments showed a peak usually near $G=2 e^{2} / h$, and one or two hills (near $2 e^{2} n / h$ for $n=2,3$ ), that become broader with increasing $n$. The first peak, which is marked for $\mathrm{Na}, \mathrm{Al}$, and $\mathrm{Au}$ wires, indicates the higher probability of the single ballistic channel being operational shortly before the break of the neck. Surprisingly, such a peak is reported ${ }^{11-13}$ to be absent in ferromagnetic metal contacts and wires such as $\mathrm{Ni}, \mathrm{Co}, \mathrm{Fe}$. This situation is interpreted as the absence of conductance quantization. ${ }^{13}$ Hansen et al. ${ }^{13}$ suggested that the absence of quantization is related to the impurities in the nanowires since transition metals are relatively more reactive. The observation that $D(G)$ increases in amplitude as $G$ decreases below $2 e^{2} / h$, is presented as a supporting argument. They also pointed out that the absence of quantization may be due to the complicated electronic structure that deviates strongly from the nearly free electron picture. Furthermore the conductance of the atomic-scale contact of the $\mathrm{Ni}$ tip on the $\mathrm{Ni}$ substrate (which is measured by the jump to contact process ${ }^{14}$ ) revealed a $D(G)$ that was rather dispersed and showed no relevant peaks near multiples of $e^{2} / h$. These results strengthen the role of the complex electronic structure in affecting $D(G)$, since these experiments were performed in both He gas atmosphere and in high vacuum. Earlier, based on the formalism developed for quantum point contact, it was predicted ${ }^{3}$ that the plateaus should not occur in certain constrictions since the tunneling contribution becomes important owing to several states close to $\mathrm{E}_{F}$.

This paper, which is complementary to our earlier work, ${ }^{3,8}$ proposes a mechanism to explain the absence of the peaks in $D(G)$ obtained from the constrictions produced in transition-metal wires. To this end, we consider only the last stage of stretching of the nanowire or the initial stage of an atomic contact where a neck consisting of a single $\mathrm{Ni}$ atom is connected to the right and left $\mathrm{Ni}$ electrodes as described schematically by the inset in Fig. 1. Ni displays the following properties which are markedly different from the metals such as $\mathrm{Na}, \mathrm{Al}$ and $\mathrm{Au}$ :

(i) $\mathrm{Ni}$ is an open-shell atom with the $3 d^{8} 4 s^{2}$ ground state configuration. In the crystalline state, the $4 s$ band is $\sim 7 \mathrm{eV}$ wide, and is half filled. The narrow $3 d$ bands (that have a width of 3-4 eV) overlap with both the $4 s$ band and the Fermi level. ${ }^{15}$ As a result, there are bands close to $\mathrm{E}_{F}$.

(ii) The $3 d$ states of the $\mathrm{Ni}$ atom(s) at the neck (which would be fivefold degenerate when they are free) split. The splittings are derived from the nonuniform crystal field $V_{C, R}$ and $V_{C, L}$, of the right and left electrodes, respectively. The corresponding hopping matrix element, 

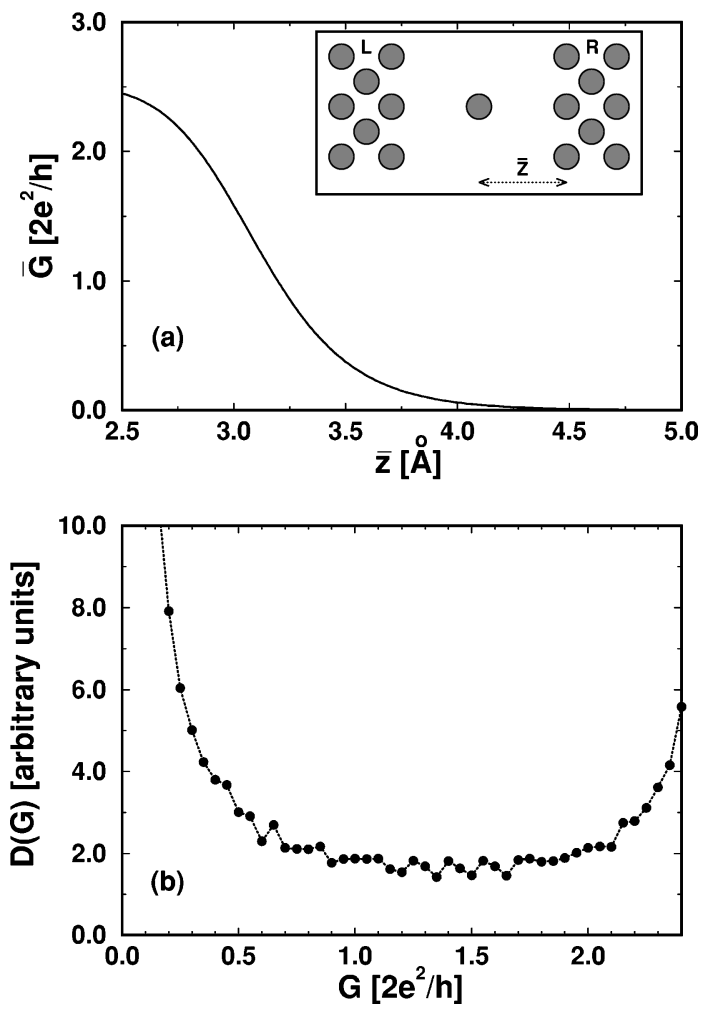

FIG. 1. (a) The variation of the conductance through a single atom neck with separation $\bar{z}$. The model for the neck is described by the inset with filled circles showing $\mathrm{Ni}$ atoms ( $L$ and $R$ are left and right electrodes, respectively. (b) The distribution of the conductance $D(G)$.

$$
V_{e n}(\bar{z})=\left\langle\varphi_{e}\left|-\frac{\hbar^{2}}{2 m} \nabla^{2}+V_{C, R}(\vec{r})+V_{C, L}(\vec{r})+V_{0}(\vec{r})\right| \varphi_{d n}\right\rangle
$$

between the electrode state $\varphi_{e}$ and a particular $3 d$ state $\varphi_{d n}$ of the neck atom depends on the symmetry of the latter. Here, $V_{o}(\vec{r})$ is the potential due to the $\mathrm{Ni}$ atom at the neck with distance $\bar{z}$, from the electrode surfaces. Consequently, the presence of $\mathrm{Ni}$ atom(s) at the neck gives rise to several closely lying energy eigenstates $\epsilon_{n}$ near $E_{F}$. Their splitting $\Delta \epsilon_{n}=\epsilon_{n+1}-\epsilon_{n}$ depends on $V_{e n}(\bar{z})$ (and also on the atomic configuration at the neck). $\epsilon_{n}$ 's become even more densely and closely distributed near $\mathrm{E}_{F}$ if the neck incorporates several (more than one) $\mathrm{Ni}$ atoms. Furthermore $\epsilon_{n}$ 's shift and hence $\Delta \epsilon_{n}(\epsilon)$ fluctuate significantly in the course of stretching of the neck where $\mathrm{Ni}$ atoms are displaced by changing their relative positions. Note that $\bar{z}$ normally increases with increasing $s$.

(iii) The Ni crystal is a ferromagnetic material with the Curie temperature $T_{c}=631 \mathrm{~K}$. For $T<T_{c}$, the permanent magnetization owing to the exchange (or Weiss) field $H_{E}$ lifts the spin degeneracy of the states of $\mathrm{Ni}$ atoms at the neck. Assuming single domain electrodes, the resulting splitting $\Delta \epsilon\left(H_{E}\right)=\epsilon_{n \downarrow}\left(H_{E}\right)-\epsilon_{n \uparrow}\left(H_{E}\right)$ is estimated to be $\mu_{B} H_{E}$ $\sim 0.1 \mathrm{eV}$. As a result, the number of states is doubled (decoupled) due to the ferromagnetic interaction; each state, $\varphi_{n \downarrow}$ or $\varphi_{n \uparrow}$, can form a distinct channel at $E_{F}$ having maximum conductance $e^{2} / h$. (iv) At room temperature the permanent magnetic field $H_{E}$ fluctuates due to the spin wave fluctuations. This, in turn, leads to fluctuation in the electronic energy spectrum $\epsilon_{n \downarrow, \uparrow}$ and also in level spacing. The corresponding fluctuation of energy $|\delta \epsilon|$ is calculated to be $\sim 0.01 \mathrm{eV}$.

Based on the above discussion, a connective neck of $\mathrm{Ni}$ (as well as $\mathrm{Fe}$ and $\mathrm{Co}$ ) can be described by a short constriction that has several closely lying states near $E_{F}$. Their energies and level spacing vary significantly in the course of stretching of the wire. We estimate the average level spacing $<\Delta \epsilon_{n}>\sim 0.1 \mathrm{eV}$. Ideally, each state at $E_{F}$ can form a current transporting state and hence appears as a channel contributing to $G$ at most $e^{2} / h$. One state becoming farther away from $E_{F}$ shall give rise to a fall in the $G$ versus $s$ curve. However, the tunneling through a number of closely spaced states near $E_{F}$ hinders the plateaus of $G(s)$. This situation is examined by a model ${ }^{16}$ whereby the $\mathrm{Ni}$ atom(s) between two Ni electrodes is (are) represented by a tier of states between two quasicontinuous metallic state distributions. Each state $\epsilon_{n, \sigma}$ is broadened and becomes a resonance with Lorentzian $\rho_{n, \sigma}(\epsilon)$ distribution ${ }^{17}$ centered at $\epsilon=\epsilon_{n, \sigma}$ $+\Lambda, \Lambda$ being a small shift. The half-width at half-maximum is $\Gamma \approx \pi\left|V_{e n}\right|^{2}$. According to Kalmeyer-Laughlin, ${ }^{18}$ the conductance due to the state $\epsilon_{n, \sigma}$ near $E_{F}$ is proportional to $\rho_{n, \sigma}\left(E_{F}\right) / \rho_{n, \sigma}\left(\epsilon_{n, \sigma}+\Lambda\right)$. Thus $G \sim G_{r e s} \Gamma^{2}\left[\left(E_{F}-\epsilon_{n \sigma}-\Lambda\right)^{2}\right.$ $\left.+\Gamma^{2}\right]^{-1}$ with the maximum resonance conductance ${ }^{19} G_{\text {res }}$ $\sim e^{2} / h$. We approximated the electrode wave function $\varphi_{e}$ by linear combination of $\varphi_{4 s}(r)$ and $\varphi_{3 d}(r) \mathrm{Ni}$ orbitals according to their bulk population with the radial wave functions parametrized by Clementi and Roetti. ${ }^{20}$ Interestingly, the exponent $\xi$ of the tail of $\varphi_{e}$ (approximated by $e^{-\xi z}$ for $z$ $>2.2 \AA)$ is found to be in reasonable agreement with the relation $\xi \sim \sqrt{\left(2 m / \hbar^{2}\right) \Phi}$. ( $\Phi$ is the work function of Ni determined experimentally). The matrix element $V_{e n, \sigma}$ of the state $\epsilon_{n, \sigma}(\bar{z})$ corresponding to the separation $\bar{z}$ is calculated within the Hückel approximation,

$$
V_{e n, \sigma}(\bar{z})=\kappa\left\langle\varphi_{e}(\vec{r}) \mid \varphi_{d n}\left(\vec{r}^{\prime}\right)\right\rangle \frac{E_{F}+\epsilon_{n, \sigma}}{2}
$$

Here $\kappa$ is taken to be 1.7 and the overlap matrix is expressed by the two-center integrals between $\varphi_{d n}$ and $\varphi_{e}$ located at the nearest electrode atom. While the Ni atoms are normally absorbed at the hollow site of Ni surfaces, the Ni atom at the neck is taken at the top site as described in Fig. 1. This configuration is in agreement with the atomic simulations ${ }^{8}$ indicating the fact that the top site is favored just before the break of the wire under uniaxial tensile stress. We found that $\left\langle\varphi_{e}(\vec{r}) \mid \varphi_{d n}(\vec{r})\right\rangle$ decays exponentially with separation $\bar{z}$ with the decay constant $\alpha=-1.367 \AA^{-1}$. As clarified above, the value of $\epsilon_{n, \sigma}$ and its variation with $\bar{z}$ (or $s$ in the experiment) is rather random. This situation is taken into account as follows: We assumed five states near $E_{F}$ contributing to the conductivity with their level spacings $\Delta \epsilon$ are taken as random variable (changing between $0-0.3 \mathrm{eV}$ ) at $\bar{z}=2.2 \AA$. Furthermore, we assumed that these energies converge linearly to an energy $\epsilon_{3 d}=E_{F}-\Delta^{\prime}$ as the neck is stretched 
from $\bar{z}=2.2 \AA$ to $\bar{z}=5 \AA$. In consideration of the groundstate configuration and excited states near the ground state ${ }^{21}$ of the $\mathrm{Ni}$ atom, $\Delta^{\prime}$ is also taken as a random variable with $3<\Delta^{\prime}<4 \mathrm{eV}$. We calculated the conductance by using 5000 different random variables. The variation of the conductance obtained for their average value $\bar{G}(\bar{z})$ and the conductance distribution $D(G)$ obtained from $5000 G(\bar{z})$ are shown in Figs. 1(a) and 1(b), respectively. The plateaulike behavior of $\bar{G}(\bar{z})$ for $\bar{z} \sim 2.5 \AA$ and the corresponding rise of $D(G)$ for $G>4 e^{2} / h$ is related with the initial condition. Due to the absence of plateaus in $\bar{G}(\bar{z})$, no peak appears in $D(G)$ curve. Note that the $D(G)$ curve in Fig. 1 is similar to the experimental curve reported in Ref. 13. This demonstrates that the complex electronic structure near $E_{F}$ is responsible from the experimentally observed behavior of $D(G)$. We also point out that the span of random variables and number of $\epsilon_{n \sigma}$ participating in conductance affects the value of $\bar{G}(\bar{z})$, but not the behavior of $D(G)$.

In conclusion, we showed that owing to the closely spaced spin-split states near $E_{F}$ at the neck, the tunneling smears out the plateaus in $G(s)$ curve of ferromagnetic nanowires. ${ }^{22}$ As a result, the $D(G)$ curve does not display marked peaks.

We acknowledge a helpful discussion with Professor Ş. Süzer. We also thank M. Brandbyge and L. Olesen for sending us their Ph.D. theses.
${ }^{1}$ J. K. Gimzewski and R. Möller, Phys. Rev. B 36, 1284 (1987).

${ }^{2}$ N. Agrait, J. G. Rodrigo, and S. Vieira, Phys. Rev. B 47, 12345 (1993); J. I. Pascual, J. Mendéz, J. Gómez-Herrero, A. M. Baró, N. Garcia, and V. Thien Binh, Phys. Rev. Lett. 71, 1852 (1993).

${ }^{3}$ S. Ciraci and E. Tekman, Phys. Rev. B 40, 11969 (1989).

${ }^{4}$ T. N. Todorov and A. P. Sutton, Phys. Rev. Lett. 70, 2138 (1993); A. M. Bratkovsky, A. P. Sutton, and T. N. Todorov, Phys. Rev. B 52, 5036 (1995); T. N. Todorov and A. P. Sutton, ibid. 54, R14 234 (1996).

${ }^{5}$ N. D. Lang, Phys. Rev. B 52, 5335 (1995); A. Yazdani, D. M. Eigler, and N. D. Lang, Science 272, 1921 (1995); N. D. Lang, Phys. Rev. Lett. 79, 1357 (1997).

${ }^{6}$ H. Mehrez, S. Ciraci, A. Buldum, and Inder P. Batra, Phys. Rev. B 55, R1981 (1997).

${ }^{7}$ E. Tekman and S. Ciraci, Phys. Rev. B 43, 7145 (1991).

${ }^{8}$ H. Mehrez and S. Ciraci, Phys. Rev. B 56, 12632 (1997); H. Mehrez, S. Ciraci, C. Y. Fong, and Ş. Erkoç, J. Phys.: Condens. Matter 9, 10843 (1997).

${ }^{9}$ A. Buldum, S. Ciraci, and Inder P. Batra, Phys. Rev. B 57, 2468 (1998); For dipole and resonant excitations see, for example, A. Buldum and S. Ciraci, ibid. 54, 2175 (1996) and references therein.

${ }^{10}$ G. Rubio, N. Agraï and S. Vieira, Phys. Rev. Lett. 76, 2302 (1996).

${ }^{11}$ L. Olesen, Ph.D. thesis, Institute of Physics and Astronomy, University of Aarhus, 1996.

${ }^{12}$ M. Brandbyge, Ph.D. thesis, Center for Atomic-Scale Materials Physics and Technical University of Denmark, 1997.
${ }^{13}$ K. Hansen, E. Laegsgaard, I. Stengaard, and F. Besenbacher, Phys. Rev. B 56, 2208 (1997).

${ }^{14}$ C. Sirvent, J. G. Rodrigo, S. Vieira, L. Jurczyszyn, N. Mingo, and F. Flores, Phys. Rev. B 53, 16086 (1996).

${ }^{15}$ See, for example, E. I. Zornberg, Phys. Rev. B 1, 244 (1970).

${ }^{16}$ A rigorous treatment of the conductance of stretching Ni neck requires the evaluation of the current operator with electronic states calculated self-consistently depending on the detailed position of Ni ions. The objective of the present study is, however, to show that the tunneling destroys the staircase structure of $G(s)$.

${ }^{17}$ P. W. Anderson, Phys. Rev. 124, 41 (1961). D. M. Newns, Phys. Rev. 178, 1123 (1969).

${ }^{18}$ V. Kalmeyer and R. B. Laughlin, Phys. Rev. B 35, 9805 (1987).

${ }^{19}$ Owing to the backscattering $G_{\text {res }}$ can be smaller than $e^{2} / h$ (see Ref. 7). Moreover, $G_{\text {res }}$ may depend on the symmetry of the state $\varphi_{d n}$. In the calculation we take uniform $G_{r e s}$, which is in fact an unfavorable situation for the present theory.

${ }^{20}$ E. Clementi and C. Roetti, At. Data Nucl. Data Tables At. Data Nucl. Data Tables 14, 177 (1974).

21 J. M. Dyke, B. W. J. Gravenor, R. A. Lewis, and A. Morris, J. Phys. B 15, 4523 (1982).

${ }^{22}$ Alternatively, the effect of tunneling can be treated within adiabatic approximation. The tier of the states $\epsilon_{n, \sigma}$ is transformed to a tier of effective potential $\epsilon_{n, \sigma}(z)$. As $\bar{z}$ increases $\epsilon_{n, \sigma}(\bar{z})$ is lowered. The level spacings and their position relative to $E_{F}$ vary with $s$. The tunneling and related conductance can be calculated by Wentzel-Kramers-Brillouin approximation. 